\title{
COMPLICACIONES DEL PARTO VAGINAL EN HOSPITALES PROVISIONALES POST TERREMOTO EN ICA, PERÚ.
}

\author{
Judith Choque-Miranda ${ }^{(1,2)}$, Erika Facundo-Chacaltana ${ }^{(1,2)}$, \\ Franci Pimentel-De la cruz ${ }^{(1,2)}$, Marjorie Ybaseta-Soto ${ }^{(1,2)}$
}

1. Facultad de Medicina, Universidad Nacional San Luis Gonzaga. Ica, Perú.

2. Médico Cirujano

\section{RESUMEN}

Objetivo: Determinar y comparar la frecuencia de complicaciones del parto en hospitales provisionales post terremoto en Ica. Material $\boldsymbol{y}$ métodos: Se realizó un estudio descriptivo entre junio y diciembre de 2011 . En 07 meses se atendieron 665 partos en el Hospital Provisional del Hospital Santa María del Socorro (HSMSI) y 1,012 en el Hospital Provisional del Hospital Regional de Ica (HRI). Resultados: Treinta y seis pacientes atendidas en el HSMS y 34 en el HRI, tuvieron complicaciones. La frecuencia global de complicaciones maternas del parto fue $5,5 \%$ en el HSMS y $3,5 \%$ en el HRI. Se encontró diferencia estadísticamente significativa $(p<0,05)$ para ambas sedes en las siguientes variables: Edad materna menor de 20 años, primiparidad, multiparidad y personal que atendió el parto. Conclusión: Se encontró una alta frecuencia de las complicaciones en el período expulsivo y que es de necesidad la capacitación y supervisión continua a internos de obstetricia, internos de medicina y residentes de la especialidad durante la atención del parto vaginal.

PALABRAS CLAVE: Complicaciones del trabajo de parto, Paridad, Obstetriz. (fuente: DeCS BIREME)

\section{COMPLICATIONS FROM A VAGINAL CHILBIRTH IN PROVISIONAL HOSPITALS POST EARTHQUAKE IN ICA, PERU}

\section{ABSTRACT}

Objective: To determine and compare the frequency of complications of childbirth in provisional hospitals post-earthquake in Ica, during June to December 2011. Material and methods: It was realized a descriptive study. In 07 months there were 665 childbirths in the Provisional hospital of the Santa Maria del Socorro Hospital (HSMSI) and 1.012 births in the provisional Hospital of the Regional Hospital of Ica (HRI) Results: Thirty six patients were attended in the HSMS and 34 in the HRI, had complications. The global frequency of maternal complications of childbirth was $5.5 \%$ in the HSMS and $3.5 \%$ in the HRI.It was found Statistically significant difference ( $p<0.05)$ for both sites in the following variables: maternal age younger than 20 years, primiparity, multiparity and personal who attended the childbirth.

Conclusions: It was found a high frequency of complications in the expulsive period and that is of necessity the training and supervision continues to the internal of obstetrics, internal of medicine and residents of the speciality during the attention of the vaginal childbirth.

KEY WORDS: Obstetric Labor Complications, Parity, Midwives. (source: MeSH NLM).

\section{INTRODUCCIÓN}

La salud materna tiene una importancia ampliamente reconocida en nuestro país y el mundo en especial en América Latina. Este reconocimiento ha llevado a un esfuerzo constante para mejorar las áreas de la salud involucradas, pero aún son insuficientes, pues los indicadores de morbilidad y de mortalidad materna no han mejorado mucho ${ }^{(1,2)}$. El embarazo, a cualquier edad, constituye un hecho biopsicosocial de gran trascendencia; en la adolescencia cobra mayor importancia por los riesgos que puede conllevar para del binomio madre-hijo, además puede producir complicaciones invalidantes definitivas para el futuro en los planos orgánico, social y psicológico de las madres
En el Perú, cientos de mujeres pierden la vida anualmente sobre todo en las zonas rurales y en los cinturones de pobreza de las ciudades, ya sea durante el embarazo, en el parto o el puerperio, como consecuencia de su pobreza, analfabetismo, bajo nivel de instrucción, escasos niveles de ingreso, marginación social, deplorable estado de salud y limitado acceso a los servicios de salud ${ }^{(3,4,5,6,7)}$.

Las complicaciones maternas más frecuentes son las hemorragias Post-Parto siendo las causas: Atonía Uterina $(90 \%)$, desgarros o rupturas del tracto genital $(6 \%)$ y la retención de restos placentarios (4\%) 
Avila A. $(2,004)^{(16)}$ en su estudio de complicaciones del parto reporta una incidencia de $13,25 \%$ de desgarros vulvoperineales estudio realizado en el Hospital Santa Rosa. Valdez S. $(2,000)^{(17)}$ reportó una incidencia de $22.2 \%$ de complicaciones maternas en el hospital municipal de Santa Cruz del Sur, siendo más frecuentes entre adolescentes llegando a $42.4 \%$ del total. Según J. Ybaseta $(1,998)$ en su estudio sobre complicaciones maternas en los Hospitales del Ministerio de Salud de Ica, encontró que la complicación global del parto vaginal en el HSMSI fue de $10.20 \%$ mientras que el HRI llegó al $6.48 \%$ habiendo una diferencia estadísticamente significativa; en ambos hospitales existía una alta incidencia de complicaciones del parto en el período expulsivo.

Conociendo que las complicaciones del parto, son causa de gran morbimortalidad materna, se hace necesario determinar y comparar la frecuencia de complicaciones del parto en hospitales provisionales post terremoto en Ica.

\section{MATERIAL Y MÉTODOS}

Fue un estudio descriptivo, comparativo, observacional realizado en los Hospitales provisionales del HSMS Y HRI pertenecientes al Ministerio de Salud en la provincia de Ica, Perú post terremoto. Ocurrido el mismo, los hospitales fueron instalados en ambientes provisionales, uno en una fábrica de bebidas y el otro en un campo deportivo del Instituto Peruano del Deporte, Disminuyendo, en ambas Instituciones, a menos del $50 \%$ la capacidad resolutiva en relación a antes del desastre.

La investigación abarcó a toda la población de parturientas atendidas en el HSMS y HRI las cuales fueron 665 y 1012 respectivamente, durante el período comprendido entre el 01 de Junio al 31 de Diciembre del 2011. Se excluyeron todas las pacientes cuyo parto no fue institucional, o que fueron admitidas por presentar complicaciones durante cualquier período del parto, o cuando la vía fue abdominal (cesárea).

Se obtuvieron los números de las historias clínicas de las parturientas de los libros de sala de partos y sala de operaciones de las pacientes que presentaron complicaciones maternas en el periodo expulsivo, alumbramiento y puerperio inmediato; se revisaron las historias clínicas en el departamento de estadística. La información fue registrada en una ficha pre-elaborada con las siguientes variables en estudio: edad materna, edad gestacional por fecha de última menstruación, paridad, gravidez, periodo intergenésico, control prenatal, complicaciones durante el expulsivo, complicaciones durante el alumbramiento y el puerperio inmediato.

El contenido de las hojas de recolección fue ingresado a una base de datos construida con el programa
Microsoft Excel. Para el procesamiento y análisis estadístico se utilizó el programa EPI INFO versión 6.04 de la OMS, para investigaciones en salud. Se revisó la base de datos, para el control de calidad de la información, buscando errores de digitación, duplicación, omisión de datos o datos aberrantes. Se calcularon las frecuencias, porcentajes y la prueba Ji cuadrado para determinar la significancia estadística eligiendo un nivel de $p<0,05$.

El estudio contó con la autorización de las autoridades competentes.

\section{RESULTADOS}

Treinta y seis pacientes del HSMS y 34 del HRI tuvieron complicaciones maternas en el periodo expulsivo, alumbramiento y puerperio, que constituyen el $5,5 \%$ y el $3,5 \%$ respectivamente $(p<0,05)$.

En el $50 \%$ las complicaciones ocurrieron en el periodo expulsivo en el HSMS y en el $35.29 \%$ en el HRI. En el alumbramiento, el $\mathrm{HRI}$ tuvo mayor incidencia de complicaciones (58.82\%) que el HSMS (38.89\%). En el puerperio inmediato los porcentajes fueron $11.11 \%$ para el HSMS y $5.88 \%$ para el HRI ( $p>0.05)$.

Tabla 1. Características de los pacientes

\begin{tabular}{lccccc}
\hline Variable & HSMSI & HRI & & \\
& $\mathrm{n}$ & $\%$ & $\mathrm{n}$ & $\%$ & $\mathrm{p}$ \\
Grupo etario & & & & & \\
$<$ < 20 años & 4 & 11 & 7 & 22 & $<0,05$ \\
20 a 35 años & 28 & 78 & 23 & 68 & \\
>s 35 años & 4 & 11 & 3 & 10 & \\
Paridad & & & & & \\
Primípara & 13 & 37 & 19 & 57 & $<0,05$ \\
Multípara & 19 & 51 & 12 & 35 & $<0,05$ \\
Gran multíp. & 4 & 12 & 3 & 8 & \\
Control prenatal & & & & & \\
Sin control & 14 & 39 & 10 & 31 & $>0,05$ \\
Inadecuado & 13 & 38 & 14 & 38 & $>0,05$ \\
Adecuado & 9 & 23 & 10 & 31 & $>0,05$ \\
\hline
\end{tabular}


Tabla 2. Complicación por periodo del parto

\begin{tabular}{|c|c|c|c|c|}
\hline Tipo de comp. & \multicolumn{2}{|c|}{ HSMSI } & \multicolumn{2}{|c|}{ HRI } \\
\hline \multicolumn{5}{|l|}{$\begin{array}{l}\text { Periodo } \\
\text { expulsivo }\end{array}$} \\
\hline $\begin{array}{l}\text { Desgarro Cervical } \\
\text { Desg. Mucosa }\end{array}$ & 2 & 5,56 & 1 & 2,94 \\
\hline $\begin{array}{l}\text { Vaginal } \\
\text { Desg. }\end{array}$ & 2 & 5,56 & 3 & 8,82 \\
\hline $\begin{array}{l}\text { Vulvoperineal I } \\
\text { Desg. }\end{array}$ & 9 & 25 & 5 & 14,71 \\
\hline $\begin{array}{l}\text { Vulvoperinel II } \\
\text { Desg. }\end{array}$ & 3 & 8,33 & 2 & 5,88 \\
\hline $\begin{array}{l}\text { Vulvoperineal III } \\
\text { Desg. }\end{array}$ & 1 & 2,78 & 1 & 2,74 \\
\hline $\begin{array}{l}\text { Vulvoperineal IV } \\
\text { Periodo } \\
\text { alumbramiento } \\
\text { Retención de }\end{array}$ & 1 & 2,78 & 0 & 0 \\
\hline $\begin{array}{l}\text { placenta } \\
\text { Ret. Restos }\end{array}$ & 3 & 8,33 & 1 & 3,57 \\
\hline $\begin{array}{l}\text { placentarios } \\
\text { Ret. Memb. }\end{array}$ & 7 & 19,44 & 2 & 7,14 \\
\hline Placentarias & 4 & 11,11 & 11 & 39,29 \\
\hline \multicolumn{5}{|c|}{ Puerperio inmediato } \\
\hline $\begin{array}{l}\text { Atonía uterina } \\
\text { Hemorragia }\end{array}$ & 3 & 8,33 & 1 & 3,57 \\
\hline puerperal & 7 & 19,44 & 2 & 7,14 \\
\hline Hematoma vulvar & 4 & 11,11 & 11 & 39,29 \\
\hline
\end{tabular}

En el HSMS las pacientes con complicaciones fueron atendidas por obstetrices en el $94 \%$ y por médicos en $5 \%$, mientras que en el HRI el grupo de complicadas fue atendido por médicos en el $29 \%$ y por obstetrices en el $70 \%$. En ambos hospitales la mayor parte del grupo de las pacientes complicadas fueron atendidas por obstetrices, aunque en el HRI hubo una mayor proporción de pacientes de este grupo atendidas por médicos. Estas diferencias fueron estadísticamente significativas.

\section{DISCUSIÓN:}

La frecuencia global de complicaciones maternas del período expulsivo, alumbramiento y puerperio fue de $5,5 \%$ para el HSMS y $3,5 \%$ para el $\mathrm{HRI}$, siendo porcentajes inferiores a los encontrados en otras investigaciones revisadas ${ }^{(17,18)}$.

En este estudio se encontró una mayor frecuencia de complicaciones maternas entre los grupos etarios de 20 a 35 años, en ambos hospitales. PÉREZ ${ }^{(1)}$, refiere que, el embarazo en los extremos de la vida reproductiva es una situación condicionante de alto riesgo obstétrico y perinatal. BOTERO ${ }^{(10)}$, informa que se ha demostrado que la edad comprendida entre 20 y 29 años es la mejor para el proceso reproductivo y además es el grupo con menos mortalidad perinatal. Se sabe que al aumentar la edad también se incrementa la posibilidad de sufrir enfermedades crónicas que agravan la salud de la madre. En nuestro caso La mayor incidencia de complicaciones maternas se produjo entre las mujeres de 20 a 35 años, en ambos establecimientos, esto tal vez sea porque el mayor porcentaje de partos se da en este grupo etario.

La mayor incidencia de complicaciones maternas en el HSMS fue entre multíparas y en el HRI las afectadas fueron las primíparas. MONGRUT ${ }^{(8)}$, describe la primiparidad (con menos de 15 años y más de 35 años) y la multiparidad como factores de alto riesgo para presentar complicaciones relacionadas al embarazo, parto y puerperio.

SCHWARCZ ${ }^{(11)}$, refiere que en la primiparidad precoz el embarazo sorprende a la mujer con un aparato genital insuficientemente desarrollado e infantil. En el parto pueden sobrevenir distocias por falta de desarrollo pelviano y aquí, como en el alumbramiento, originarse anomalías por deficiencias de la contracción uterina. GONZÁLES y Col. ${ }^{(20)}$, refieren que las complicaciones del período expulsivo ocurren con mayor frecuencia en los órganos que no han llegado a su madurez fisiológica.

Respecto a la primípara añosa, el útero que durante años ha estado recibiendo periódica y cíclicamente influjos nerviosos y hormonales, va perdiendo su normalidad fisiológica. Las fibras musculares dejan de tener dos de sus propiedades fundamentales: elasticidad y contractilidad al sufrir degeneración fibrosa. La falta de extensibilidad del músculo uterino puede ser causa de interrupción de la gestación. En el parto y alumbramiento el útero se contrae con escasa energía (inercia, hemorragias). La articulación del cóccix se anquilosa. Las partes blandas (cuello, vagina y periné) ofrecen excesiva resistencia, porque han perdido su elasticidad y blandura, ello acarrea prolongación y detención del parto, desgarros o estallidos, hemorragias del alumbramiento, mala involución uterina e intervenciones frecuentes ${ }^{(5)}$.'

En este estudio no se encontró diferencia estadísticamente significativa en la población de parturientas del HSMS al comparar los grupos que tuvieron control prenatal adecuado con el grupo que tuvo control prenatal inadecuado y el grupo que no tuvo control prenatal. El éxito del cuidado prenatal radica en la identificación precoz y oportuna de las gestantes que tienen factores de riesgo, darles un valor clínico y planificar el manejo y la atención adecuada, para erradicar o controlar los factores de riesgo.

En esta investigación se determinó que el porcentaje de complicaciones maternas del periodo expulsivo fue mayor en relación a otros periodos no existiendo diferencia estadísticamente significativa en ambos hospitales. A diferencia de los estudios revisados donde hallan menores porcentajes de complicaciones en este periodo del parto ${ }^{(16,17,23,24,25,26,27)}$. 
En esta investigación, con respecto a las complicaciones del alumbramiento, sólo se enco. alta frecuencia en el grupo de retención de membra 13 placentarias en el HRI. VARGAS ${ }^{(28)}$, halló incidencia del $80 \%$ de retención de restos placenta.... en 120 pacientes que presentaron complicaciones. Por otro lado, existen maniobras que no debieran realizarse como la tracción excesiva del cordón umbilical que puede producir la sección traumática de éste y obliga a la extracción manual de la placenta; también puede ocasionar a una posible inversión uterina. Los masajes y compresiones del útero pueden producir contracciones localizadas, con desprendimientos parciales de la placenta y hemorragia. Pueden determinar también una contractura uterina con retracción del anillo de Bandl y retención de la masa placentaria, incluso ya desprendida.

En esta investigación se pudo observar que no hubo diferencia estadísticamente significativa, entre ambas instituciones, para las complicaciones del puerperio inmediato. La incidencia de hemorragias durante el puerperio, según BOTERO ${ }^{(10)}$ se presenta entre el 1 y $10 \%$ de puérperas; para LUDMIR $^{(14)}$, la incidencia de hemorragia post parto es elevada, la hemorragia es la principal causa de muerte intrahospitalaria en el Perú; sin embargo en los hospitales de Lima es la segunda causa de muerte materna. SIBAI ${ }^{(23)}$, encontró que las causas más importantes de hemorragia post parto fueron: atonía uterina $90 \%$, desgarros del canal $6 \%$ y retención de restos $4 \%$.

En este estudio se encontró que la mayor parte del grupo de pacientes complicadas fueron atendidas por obstetrices. YBASETA ${ }^{(18)}$, reportó que el $93 \% \mathrm{HSMSI}$ y 67,51 en el $\mathrm{HRI}$ de complicaciones maternas fueron atendidas por obstetrices, PAVON ${ }^{(24)}$, encontró mayor frecuencia de complicaciones entre pacientes atendidas por el médico interno llegando hasta un 40.4\%, determinando que la frecuencia de complicaciones estaba en relación con el número de partos que atendía.

\section{CONCLUSIÓN}

La frecuencia global de complicaciones del Parto en el HSMS fue del 5,5\% y del HRI de 3,5\% del total de partos atendidos, observándose que las complicaciones del parto vaginal en ambos hospitales es menor o similar a lo encontrado en la bibliografía revisada $(17,18,19,20)$. Siendo importante tomar medidas preventivas como capacitación y supervisión en la atención del parto.

\section{REFERENCIAS BIBLIOGRÁFICAS}

1. Pérez A. Obstetricia. 2da. Ed. Santiago de Chile. Publicaciones Técnicas Mediterráneo. 1994.
2. Climent I. Representaciones sociales sobre el embarazo y el aborto en la adolescencia: perspectiva de las adolescentes embarazadas. Cuad. Fac. Humanid. Cienc. Soc., Univ. Nac. Jujuy [online]. 2009, n.37, pp. 221-242. ISSN 1668-8104.

3. Ybaseta-Medina J. Factores de riesgo asociados a la mortalidad materna en la Región de Ica, Perú 2001-2005. Rev. méd. panacea $2011 ; 1: 9-13$.

4. Dirección Regional de Salud la Libertad. Evaluación del plan estratégico de intervención para la reducción de la mortalidad materna en la Libertad 2001 - 2003.

5. Ministerio de Salud. Mortalidad materna en el Perú. Lima Perú 2003.

6. Ministerio de Salud. Lineamientos de política sectorial para el periodo $2002-2012$. principios fundamentales para el plan estratégico sectorial del quinquenio agosto 2001, julio 2006. Lima, Perú.

7. Torres Y, Montoya L. Cuartas J, Osorio G. Factores asociados a mortalidad maternaestudio de casos y controles. Medellín 2001 2003. Rev CES Med. 2005; 19(2):19-45.

8. Mongrut-Steane A. Tratado de Obstetricia. Cuarta Edición. Lima- Perú. 2000.

9. Mongrut A. Tratado de obstetricia. 3ra. Ed. Editorial Serator. Lima - Perú. 1989.

10. Botero J. Júbiz $\mathbf{H}$, Henao G. Obstetricia y ginecología. 5ta. Ed. Editorial Carvajal S.A. Medellín - Colombia. 1994.

11. Schwarcz R. y col. Obstetricia. 4ta. Ed. Editorial El ateneo. Argentina. 1987.

12. MINSA - Proyecto Salud y Nutrición Básica. Procedimientos y protocolos de atención en salud de la mujer. Lima - Perú. 1999.

13. MINSA - OPS - PROYECTO 2000. Emergencias obstétricas y neonatales. Lima Perú. 1998.

14. Ludmmir A. Ginecología y obstetricia: Prevención diagnóstico. tratamiento. Editorial Lima CONCYTEC. Lima - Perú. 1996.

15. Cunningham F, Mac Donald. Williams obstetricia. 4ta. Ed. Editorial Masson S.A. Barcelona - España. 1996. 
16. Avila-Fabia A. Los desgarros vulvoperineales y sus factores de riesgo en gestantes atendidas en el Hospital Santa Rosa, durante los meses de enero-junio del 2003. [Tesis para optar título profesional de Licenciada en Obstetricia]. Universidad Mayor de San Marcos; 2004. 45.pp

17. Valdés S. Embarazo en la adolescencia. Incidencia, riesgos y complicaciones. Revista Mexicana 2000; 40 (14); 8-9.

18. Ybaseta-Medina J. Complicaciones maternas en hospitales del MINSA de Ica, 1998. [Tesis para optar título de Especialista en Ginecolc y Obstetricia]. Ica: Escuela de segu especialización, Universidad Nacional Luis Gonzaga; 1999. 59.pp

19. Friedmam E. y Acke D. Toma de decisiones en obstetricia. Editorial Ericka. España. 1990.

20. Pschyrembel, W. Obstetricia Práctica. 3ra. Edición. Editorial Labor S.A. Barcelona-España. 1981.
21. Vargas 0 . Complicaciones obstétricas más frecuentes en el puerperio. Hospital Alberto Sabogal Sologuren. IPPS Callao, 1995-1997. Lima, 1998.

22. Sibai B. Postpartum hemorrhage. En: Rivlin EM, Morrinson JC, Bates GW. editors Manual of Clinical Problems in Obstetrics and Gynecology Puerperium. Boston Litle Brown.

23. Pavon $\mathbf{P}$, Gogeascoechea, $\mathbf{M}$ Durán $\mathbf{L}$ y Col. Complicaciones obstétricas en un hospital de ginecología y obstetricia. Revista Médica de la Universidad Veracruzana. 2003; 3(2): 37

Recibido: 10/01/2012

Aceptado para publicación: 24/02/2012 